\title{
The Average-Risk Age Threshold for Colorectal Cancer Screening: Should It Be Lowered?
}

\author{
Thomas F. Imperiale
}

Published online: 1 March 2011

(C) Springer Science+Business Media, LLC 2011

Screening for colorectal cancer (CRC) among average-risk adults is recommended by several guidelines organizations beginning at age 50 . This age threshold was established in part because of a dramatic increase in CRC incidence during the sixth decade of life [1]. CRC screening is considered to be both effective and cost-effective in persons 50 years or older. The most recent epidemiologic data from SEER indicate that CRC incidence has continued to fall in this age group, and screening undoubtedly accounts for part of the decline.

In contrast, the CRC picture for persons younger than age 50 is not as good: CRC incidence in this age group has risen. In particular, CRC incidence has increased among persons ages 40-45 from 12 per 100,000 in 1987 to 18 per 100,000 in 2006-a $50 \%$ increase over 20 years [2]. Persons younger than age 50 account for 7-9\% of CRC diagnoses, tend to present with more advanced disease, and have a less favorable prognosis [3-5]. There are no published data on the effectiveness of CRC screening for persons younger than 50, and the only published model that considered this age group showed that one-time colonoscopy was not costeffective [6]. Nonetheless, other screening modalities such as fecal immunochemical testing might be useful. In light of

\section{T. F. Imperiale}

Division of Gastroenterology and Hepatology, Department of Medicine, Indiana University School of Medicine, Indianapolis, IN, USA

T. F. Imperiale ( $\square)$

Regenstrief Institute, Inc., 1050 Wishard Blvd,

Indianapolis, IN 46202, USA

T. F. Imperiale

Center of Excellence for Implementing Evidence-based

Medicine, Richard L. Roudebush Veterans Affairs Medical

Center, Indianapolis, IN, USA the rising incidence and poorer prognosis of $\mathrm{CRC}$ in persons under age 50, should the average-risk screening age threshold be lowered to 40 years?

Obtaining the answer to this complex question requires quantifying and weighing the benefits, risks, and costs of various screening modalities in persons aged 40-49 years, as compared with beginning CRC screening at age 50 . Simulation models would be most appropriate for addressing this issue. One piece of information required for the models is the prevalence of clinically-important neoplasia (i.e., adenocarcinoma and advanced adenomatous polyps). What does the literature tell us about this prevalence?

In this issue, Thoma and colleagues [7] report on neoplastic findings in 247 persons aged 40-49 years as compared with those of 747 persons aged 50-59. All subjects underwent first-time colonoscopy to the cecum. In addition to reporting age-related prevalence of neoplasia, the investigators report prevalence by gender, ethnicity, and body mass index (BMI). Other than the difference in age, the two groups differed in gender distribution (the proportion of women was greater in the 40-49 group) and colonoscopy indication (the proportion of screening colonoscopies was greater in the 50-59 group) - factors that would tend to have opposing effects on neoplasia prevalence.

Not surprisingly, the study found that neoplasia prevalence was higher in the older age group: $22.6 \%$ versus $12.1 \%$ for any neoplasia, and $4.95 \%$ versus $2.02 \%$ for advanced adenomas. The protective effect of younger age on neoplasia prevalence persisted after adjustment for gender, race, and BMI. As in previous studies, men were more likely to have adenomas. Of some interest was the finding that higher BMI was associated with increased risk of neoplasia independent of age group, a finding that persisted in adjusted analyses. Subgroup analyses that had advanced adenoma as the outcome were not done, probably 
due to the small sample size and low prevalence of this finding. How do these findings add to what is already known about absolute and comparative prevalence of colorectal neoplasia?

First, the study findings by Thoma and colleagues are consistent with those of previous studies from different populations that have examined neoplasia prevalence in the 40-49 year-old age group, some of which have made comparisons to other age groups (Table 1) [8-11]. Collectively, the study findings show a consistently low risk of advanced adenoma and a nearly zero risk of adenocarcinoma. These recent findings support what has been previously reported - which is reassuring-and support current guideline recommendations for beginning average-risk screening at age 50 [12]. Second, it suggests that the prevalence of advanced adenomas is higher among 50-59 year-olds than in 40-49 year-olds, a finding that has been found less consistently among prior studies, most likely because of low power to detect clinically-important differences. How do we reconcile the near-zero prevalence of CRC among these studies with the increasing incidence among persons under age 50?

One explanation is that, despite the handful of studies, the cumulative sample size is relatively small, especially when one considers population-based disease incidence. According to SEER, CRC incidence is 14 per 100,000 among persons aged 40-45 years and 28 per 100,000 among persons $45-49$ years, as compared with 56 per 100,000 among those 50-59 years [13]. Another possible explanation is that persons who are willing to undergo colonoscopy - and screening colonoscopy, in particularmay have a risk distribution that differs from (i.e., is lower than) the general population. A third possible explanation is that the rising CRC incidence is due to increased detection instead of a true increase in incidence. If this were the case, we would expect to find a higher proportion of earlier stage disease, but this has not been observed. So, on the one hand, population-based statistics indicate that disease incidence among persons under age 50 is rising. On the other hand, colonoscopy-based clinical data suggests that CRC prevalence is extremely low, though does so with certain caveats. What other information might be helpful for deciding whether and how to screen persons younger than age 50 ?

It would be useful to know how to estimate and stratify current risk for advanced neoplasia among persons younger than 50 in order to target screening to those at high risk. We already do this for persons with a positive family history: earlier screening using any of several tests is recommended for persons who have one first-degree relative (FDR) with CRC diagnosed at age 60 or older, while earlier and more aggressive screening with colonoscopy is recommended for persons with either a single FDR diagnosed with CRC prior to age 60 or two FDRs with CRCs at any age [12]. Such persons have a lifetime risk of CRC that is two- to three-fold higher than average risk. But those with a positive family history account for about $13-15 \%$ of the population [12]; most of the CRC that occurs in persons younger than 50 is unassociated with a family history of CRC.

Beyond family history, we need a way to identify persons under age 50 who are at high risk for CRC/advanced neoplasia. Unfortunately, this topic is not well-studied. In one case-control study [14], several risk factors for advanced neoplasia in persons under age 50 were identified, including having a FDR with CRC, a history of pelvic irradiation and lightest weight since age 21. Protective features from that study included living with a spouse or significant other and having had a prior sigmoidoscopy, colonoscopy, or barium enema. This study was limited by a low participation rates for both case and control groups, a small sample size that precluded doing multivariable analysis, and a lack of ability to validate these risk factors.

The data of Thoma and colleagues, along with those of others, exist in tension with population-based statistics. Given the tragedy of diagnosing advanced CRC,

Table 1 Prevalence of colorectal neoplasia: 40-49 years

\begin{tabular}{llcclc}
\hline Location, year [Reference] & Age group & Sample size & Any neoplasia (\%) & Cancer (\%) & Advanced neoplasia (\%) \\
\hline United States (2002) [8] & $40-49$ & 906 & 12.2 & 0 & 3.5 \\
& $>=50$ & 2,515 & - & 0.5 & 3.4 \\
Poland (2006) [9] & $40-49^{\mathrm{a}}$ & 7,106 & 9.5 & 0.4 & 5.9 \\
& $50-66$ & 43,042 & 14.9 & 0.9 & 2.0 \\
United States (2008) [10] & $40-49$ & 553 & 14 & 0 & 4.0 \\
& $50-59$ & 352 & 16 & 0.3 & 2.9 \\
Korea (2010) [11] & $40-49$ & 1,930 & 22.2 & 0.2 & 6.6 \\
United States (2011) [7] & $50-59$ & 2,716 & 32.8 & 0.3 & 2.02 \\
& $40-49$ & 247 & 12.1 & 0 & 7.23 \\
\hline
\end{tabular}

a All had family history of "cancer" 
particularly in younger persons, identifying risk factors for sporadic advanced neoplasia could be useful for targeting screening to the high-risk subgroup. Thoma and colleagues found that BMI and male gender were associated with increased risk for any neoplasia. Whether these factors or others, many of which have been associated with high risk for advanced neoplasia in persons 50 years or older [15, 16], may be used to identify younger persons at high risk for advanced neoplasia remains undetermined. Perhaps some combination of factors-phenotypic, proteomic, and genomic-will eventually identify persons under age 50 without a positive family history in whom early screening will be useful. Until that time, the age for beginning average-risk CRC screening should probably best remain at 50 years. One goal for screening should be to establish a more quantitative way to estimate risk for individual patients, a goal that will facilitate moving from an agebased threshold to a risk-based one.

Acknowledgments Supported in part by NIH grant: R-01 CA 104459 .

Conflicts of interest None.

\section{References}

1. Edwards BK, Ward E, Kohler, BA, Eheman C, et al. Annual report to the nation on the status of cancer, 1975-2006, featuring colorectal cancer trends and impact of interventions (risk factors, screening, and treatment) to reduce future rates. Cancer. 2010; 116:544-573.

2. Wachter K. Colorectal cancer rates up in people aged 40 to 44 . GI \& Hepatology News. 2010;4:1-4.

3. Fairley TL, Cardinez CJ, Martin J, et al. Colorectal cancer in US adults younger than 50 years of age, 1998-2001. Cancer. 2006;107:1153-1161.

4. Marble K, Banerjee S, Greenwald L. Colorectal carcinoma in young patients. J Surg Oncol. 1992;51:179-182.
5. O'Connell JB, Maggard MA, Liu JH, Etzioni DA, Livingston EH, Ko CY. Do young colon cancer patients have worse outcomes? World J Surg. 2004;28:558-562.

6. Ness RM, Holmes AM, Klein R, Dittus R. Cost-utility of onetime colonoscopic screening for colorectal cancer at various ages. Am J Gastroenterol. 2000;95:1800-1811.

7. Thoma M, Castro F, Golowala M, Chen R. The detection rate of colorectal neoplasia by colonoscopy in average-risk patients age 40-49 vs. 50-59 years. Dig Dis Sci. doi:10.1007/s10620-0111565-6.

8. Imperiale TF, Wagner DR, Lin CY, Larkin GN, Rogge JD, Ransohoff DF. Results of screening colonoscopy among persons 40-49 years of age. N Engl J Med. 2002;346:1781-1785.

9. Regula J, Rupinski M, Kraszewska E, et al. Colonoscopy in colorectal-cancer screening for detection of advanced neoplasia. N Engl J Med. 2006;355:1863-1872.

10. Rundle AG, Lebwohl B, Vogel R, Levine R, Neugut AI. Colonoscopic screening in average-risk individuals ages 40 to 49 vs. 50 to 59 years. Gastroenterology. 2008;134:1311-1315.

11. Chung SJ, Kim YS, Yang SY, et al. Prevalence and risk of colorectal adenoma in asymptomatic Koreans aged 40-49 years undergoing screening colonoscopy. J Gastro Hepatol. 2010;25: 519-525.

12. Levin B, Lieberman DA, McFarland B, Andrews KS, et al. Screening and surveillance for the early detection of colorectal cancer and adenomatous polyps, 2008: a joint guideline from the American Cancer Society, the US Multi-Society Task Force on Colorectal Cancer, and the American College of Radiology. Gastroenterology. 2008;134:1570-1595.

13. Surveillance Epidemiology and End Results. Table 6.10. Cancer of the Colon and Rectum (Invasive). http://seer.cancer.gov/csr/ 1975_2007/browse_csr.php?section=6\&page=sect_06_table.10.h.

14. Imperiale TF, Kahi CJ, Stuart JS, et al. Risk factors for advanced sporadic colorectal neoplasia in persons younger than age 50 . Cancer Detect Prev. 2008;32:33-38.

15. Lieberman DA, Prindiville S, Weiss DG, Willett W. VA Cooperative Study Group 380. Risk factors for advanced colonic neoplasia and hyperplastic polyps in asymptomatic individuals. JAMA. 2003;290:2959-2967.

16. Betes M, Munos-Navas MA, Duque JM, Angos R, Macias E, Subtil JC, et al. Use of colonoscopy as a primary screening test for colorectal cancer in average risk people. Am J Gastroenterol. 2003;98:2648-2654. 